\title{
Evaluación de tres generaciones de polinización libre en seis progenies de papa (Solanum tuberosum L.) provenientes de semilla (sexual)
}

\author{
A. Golmirzaie; F. Serquén; R. Ortíz *
}

\section{RESUMEN}

Seis familias correspondientes a tres generaciones de polinización libre fueron evaluadas para rendimiento de tubérculos por planta y por parcela, tasa de sobrevivencia de plantas y tasa de floración. Aún cuando hubo una tendencia decreciente en los registros de rendimiento por planta a medida que se avanzó en las generaciones de polinización libre (PL), se identificaron algunas progenies con un comportamiento positivo dentro de las generaciones. Se sugiere evaluar un mayor número de progenies por generación y también un mayor número de generaciones de polinización libre. Esto permitirá identificar aquellas progenies promisorias que puedan ser cultivadas por el mayor número posible de generaciones sin que sus caracteres agronómicos y reproductivos resulten afectados.

Palabras Claves Adicionales: Polinización libre, endocría, semilla sexual, generación.

\section{SUMMARY}

\section{Evaluation of Three Open Pollinated Generations in Six True Potato Seed Progenies}

Six true potato seed families from three open polünated generations were evaluated on the basis of yield per plant, yield per plot, survival rate and flowering rate. Even though there was a decreasing tendency according to the advance in OP generations for yield per plant, some progenies with positive performance within

Aceptado para publicación: Abril 30, 1990

* Fitomejoradores, Ph.D., M.Sc. y Ph.D., respectivamente. Centro Internacional de la Papa. Apartado 5969, Lima - Perú. 
OP generations were identified. It is suggested to evaluate both, a larger number of TPS progenies and OP generations. This will permit to identify those promissory progenies that can be grown for many OP generations without reduction in their agronomic and reproductive characters.

Additional Index Words: Open pollination, inbreeding, true seed,

generation.

La propagación de papa mediante semilla sexual como una alternativa al uso de tubérculo-semilla viene siendo investigada por el Centro Internacional de la Papa (CIP) desde 1977. Algunas de sus ventajas son su reducida transmisión de enfermedades y el bajo costo que representa su empleo, por el hecho de casi eliminar el costo de almacenamiento y transporte del tubérculo-semilla $(7,9)$. Sin embargo, el éxito de su uso por parte de los agricultores está condicionado al tipo de progenie que a éstos se les proporcione. Al respecto, KidaneMariam et al. $(3,4)$ señalan que los dos principales tipos de progenies que pueden considerarse para la producción de papa para consumo son los híbridos y los de polinización libre. Su selección debe basarse en criterios biológicos que incluyan rendimiento, uniformidad de tubérculos, vigor y uniformidad de plántulas y de plantas, además de la producción de grandes cantidades de semilla de buena calidad y adaptabilidad para la producción de tubérculos provenientes de plántulas.

Estudios previos indican que las progenies híbridas muestran un mejor comportamiento en comparación con las de polinización libre. Kidane-Mariam et al. (4) obtuvieron mayor rendimiento de tubérculo así como mayor uniformidad de plántula y tubérculo en progenies híbridas que en las de polinización libre. Similares resultados encontraron Macaso y Peloquin (6) y Kidane-Mariam et al. (3), con progenies híbridas provenientes de cruzamientos $4 x-2 x$. Sin embargo, estos investigadores concuerdan en señalar la factibilidad del empleo de las progenies de polinización libre; se estima que la polinización controlada representa de 10 a $20 \%$ del costo de la semilla híbrida.

En el estudio de las progenies de polinización libre, según Atlin (1), existen dos atributos que complican la predicción de su comportamiento partiendo de la información que proporcionan sus progenitores. El primero es que en especies autetraploides los contenidos alélicos de los gametos que se unen para formar la generación inicial de una variedad sintética, son dispersados en la 
población en generaciones posteriores. De este modo, las interacciones alélicas que pueden contribuir a la selección de generaciones iniciales son perdidas rápidamente aún cuando tales interacciones pueden haber sido importantes en la selección de los progenitores. El segundo atributo es que estas variedades de polinización libre son substancialmente endocriadas. En la primera generación de polinización libre esta endocría resulta de la autofecundación y en las generaciones posteriores es resultado tanto de la autofecundación como del apareamiento entre individuos emparentados. Como consecuencia hay un cierto incremento en el coeficiente de endocría y por consiguiente una reducción en el rendimiento como reportado por Krantz (5), Mendoza y Haynes (8) y Macaso (6). Sin embargo, Atlin (1) citando a Bedi (1979) y Glendinning (1976), señala que existe una variación considerable en el comportamiento de líneas individuales SI, algunas de las cuales se aproximan a los rendimientos de los mejores híbridos.

El propósito del presente trabajo fue evaluar el comportamiento de tres generaciones de polinización libre en seis progenies de semilla en el cultivo de la papa.

\section{MATERIALES Y MÉTODOS}

El presente trabajo se realizó en las Estaciones Experimentales del CIP en las localidades de Huancayo $\left(12^{\circ} 07^{\prime} \mathrm{S}\right)$ y San Ramón $\left(11^{\circ}\right.$ $\left.08^{\prime} \mathrm{S}\right)$. Se utilizaron seis progenies pertenecientes a tres generaciones de polinización libre. La semilla de las progenies correspondiente a cada generación fue obtenida en la localidad de San Ramón.

La semilla fue sembrada sobre sustrato elaborado a base de arena, musgo y tierra en proporción 1:2:1. Cuando las plántulas tuvieron aproximadamente 4 a $6 \mathrm{~cm}$ de altura se trasvasaron a recipientes de musgo comprimido llamados "jiffy-7" donde permanecieron hasta que las plantas alcanzaron una altura promedio de $15 \mathrm{~cm}$. Luego fueron transplantadas al campo espaciadas $30 \mathrm{~cm}$ entre plantas y $90 \mathrm{~cm}$ entre surcos, y con una densidad de 40 plantas por familia y por repetición.

Las condiciones en las que se condujo el experimento fueron normales, tanto en clima como en el manejo agronómico. Se registraron datos de sobrevivencia de plántulas, rendimiento de tubérculos e intensidad de floración.

El análisis estadístico se hizo independientemente para cada localidad y para cada característica. Se hizo un análisis combinado para las dos localidades. Se empleó el diseño de bloques completos al azar con arreglo factorial para el análisis de variancia. Se usó la prueba de comparación de Duncan $(\mathrm{p}=0.05)$ para la discriminación entre los tratamientos en estudio. 


\section{RESULTADOS Y DISCUSIÓN}

Los resultados sobre rendimiento de tubérculo por planta, rendimiento por parcela, porcentaje de sobrevivencia e intensidad de floración para las tres generaciones de polinización libre se presentan en la Tabla 1. Estos muestran una tendencia decreciente para rendimiento por planta en las generaciones avanzadas de polinización libre, aunque sin diferencias estadísticamente significativas. Estos resultados confirman lo reportado por Atlin (1) y Krantz (5) en cuanto al comportamiento de las progenies de polinización libre. Así mismo, es necesario señalar la tendencia positiva para el carácter rendimiento por parcela, debido a una mayor tasa de sobrevivencia de plantas. Esto indicaría una mayor capacidad de las progenies en estas generaciones, para las condiciones de estrés que el medio ambiente pueda presentar.

Tabla 1. Comparación de tres generaciones de polinización libre agrupando el efecto de seis progenies de semilla (sexual) de papa. Los datos agrupan resultados promedio por generación de todas las familias.

\begin{tabular}{ccccc}
\hline Generación & $\begin{array}{c}\text { Rdto/planta } \\
\text { (g) }\end{array}$ & $\begin{array}{c}\text { Rdto/parcela } \\
\text { (kg) }\end{array}$ & $\begin{array}{c}\text { Sobrevivencia de } \\
\text { plántulas } \\
\text { (\%) }\end{array}$ & $\begin{array}{c}\text { Tasa de } \\
\text { Floración* }\end{array}$ \\
\hline 1 & $499 \mathrm{a}^{* *}$ & $14.761 \mathrm{a}$ & $72.6^{\mathrm{a} * *}$ & $6 \mathrm{a}^{* *}$ \\
2 & $470 \mathrm{a}$ & $14.611 \mathrm{a}$ & $76.5 \mathrm{a}$ & $6 \mathrm{a}$ \\
3 & $467 \mathrm{a}$ & $15.089 \mathrm{a}$ & $81.3 \mathrm{a}$ & $6 \mathrm{a}$ \\
\hline
\end{tabular}

* Floración: 1 = baja; 9 = alta.

** Prueba de Duncan $(p=0.05)$

El comportamiento de las progenies se muestra en las Tablas 2 y 3 en las cuales se destacan las familias 375512.33 x 81M12-164, Atzimba x LTV y 377896.12 x 377904.10. Es interesante notar que algunas de estas familias muestran un comportamiento positivo a medida que se avanza en generaciones de PL, encontrándose rendimientos por planta superiores a los obtenidos en la primera generación. Esto sugiere la necesidad de evaluar un mayor número de progenies con lo cual se incrementaría la posibilidad de encontrar progenies con un comportamiento similar al observado en este trabajo. 
Tabla 2. Comparación de seis progenies provenientes de semilla (sexual) de papa mantenidas por polinización libre (PL) en tres generaciones sucesivas. Los datos indican resultados promedio por familia y tres generaciones de PL.

\begin{tabular}{|c|c|c|c|c|}
\hline Progenie & $\begin{array}{l}\text { Rdto/parcela } \\
\text { (g) }\end{array}$ & $\begin{array}{c}\text { Sobrevivencia } \\
\text { (kg) }\end{array}$ & $(\%)$ & $\begin{array}{c}\text { Tasa de } \\
\text { Floración* }\end{array}$ \\
\hline 375512.33 x 81M12.164 & $553 a^{* *}$ & $16.800 \mathrm{a}^{* *}$ & $75.0 \mathrm{a}$ & $7 a^{*}$ \\
\hline Atzimba x LT-7 & $535 a$ & $16.411 \mathrm{a}$ & $76.7 \mathrm{a}$ & $7 a$ \\
\hline $377896.12 \times 377904.10$ & $502 \mathrm{ab}$ & $16.044 \mathrm{ab}$ & 80.3a & $8 a$ \\
\hline 7.24 PVX x DTO-28 & $437 \mathrm{bc}$ & 13.489 bc & $77.5 \mathrm{a}$ & $4 \mathrm{~b}$ \\
\hline 377895.12 x 377904.10 & 433 bc & $13.356 \mathrm{c}$ & $75.6 \mathrm{a}$ & $6 a b$ \\
\hline 103.127 SRLL x 377904.10 & $411 \mathrm{c}$ & $12.822 \mathrm{c}$ & $75.8 \mathrm{a}$ & $3 \mathrm{~b}$ \\
\hline
\end{tabular}

* Floración: 1 = baja; 9 = alta.

** Prueba de Duncan $(p=0.05)$

Tabla 3. Comparación de seis progenies provenientes de semilla (sexual) de papa mantenidas por polinización libre (PL) en tres generaciones sucesivas. Datos discriminados por progenie y por generación.

\begin{tabular}{|c|c|c|c|c|c|}
\hline Progenie & $\begin{array}{l}\text { Genera- } \\
\text { ción de } \\
\text { PL }\end{array}$ & $\begin{array}{l}\text { Rdto/ } \\
\text { planta } \\
\text { (g) }\end{array}$ & $\begin{array}{l}\text { Rdto/ } \\
\text { parcela } \\
\text { (kg) }\end{array}$ & $\begin{array}{l}\text { Sobre- } \\
\text { vivenc. } \\
\text { (\%) }\end{array}$ & $\begin{array}{l}\text { Tasa de } \\
\text { Flora- } \\
\text { ción* }\end{array}$ \\
\hline \multirow[t]{2}{*}{ Atzimba x LT-7 } & 1 & $484 a^{* *}$ & $12.967 \mathrm{~b}$ & $63.8 \mathrm{a}$ & $6 a^{* *}$ \\
\hline & $\begin{array}{l}2 \\
3\end{array}$ & $\begin{array}{l}617 a \\
505 a\end{array}$ & $\begin{array}{l}19.667 \mathrm{a} \\
16.600 \mathrm{a} b\end{array}$ & $\begin{array}{l}80.0 a \\
81.7 a\end{array}$ & $\begin{array}{l}7 a \\
7 a\end{array}$ \\
\hline \multirow[t]{2}{*}{378995.12 x 377904.10} & 1 & $504 a$ & 16.667 a & $82.5 a$ & $6 a$ \\
\hline & $\begin{array}{l}2 \\
3\end{array}$ & $\begin{array}{l}355 a \\
441 a\end{array}$ & $\begin{array}{l}10.467 \mathrm{~b} \\
12.933 \mathrm{a} b\end{array}$ & $\begin{array}{l}70.8 \mathrm{a} \\
73.3 \mathrm{a}\end{array}$ & $\begin{array}{l}4 \mathrm{~b} \\
5 \mathrm{ab}\end{array}$ \\
\hline \multirow[t]{2}{*}{377896.12 x 377904.10} & 1 & 499a & $14.600 \mathrm{a}$ & 73.3a & $6 a$ \\
\hline & $\begin{array}{l}2 \\
3\end{array}$ & $\begin{array}{l}484 a \\
523 a\end{array}$ & $\begin{array}{l}16.733 a \\
16.800 \text { a }\end{array}$ & $\begin{array}{l}86.7 a \\
80.8 a\end{array}$ & $\begin{array}{l}7 a \\
6 a\end{array}$ \\
\hline \multirow[t]{2}{*}{ 7.24 PVX x DTO-28 } & 1 & $435 a$ & 13.333 a & $76.7 \mathrm{a}$ & $4 a$ \\
\hline & $\begin{array}{l}2 \\
3\end{array}$ & $\begin{array}{l}425 a \\
450 a\end{array}$ & $\begin{array}{l}13.267 \mathrm{a} \\
13.867 \mathrm{a}\end{array}$ & $\begin{array}{l}79.2 \mathrm{a} \\
76.7 \mathrm{a}\end{array}$ & $\begin{array}{l}4 a \\
4 a\end{array}$ \\
\hline \multirow[t]{2}{*}{ 103.127 SRLL x 377904.10} & 1 & $389 a$ & 10.467 a & $59.2 \mathrm{~b}$ & $8 a$ \\
\hline & $\begin{array}{l}2 \\
3\end{array}$ & $\begin{array}{l}485 a \\
361 a\end{array}$ & $\begin{array}{l}15.000 \mathrm{a} \\
13.000 \mathrm{a}\end{array}$ & $\begin{array}{l}77.5 \text { a b } \\
90.8 a\end{array}$ & $\begin{array}{l}6 a b \\
5 \mathrm{~b}\end{array}$ \\
\hline \multirow[t]{2}{*}{375512.33 x 81M12.164 } & 1 & $686 a$ & 20.533 a & $75.8 \mathrm{a}$ & $8 a$ \\
\hline & $\begin{array}{l}2 \\
3\end{array}$ & $\begin{array}{l}453 a \\
520 a\end{array}$ & $\begin{array}{l}12.533 \mathrm{a} \\
17.333 \mathrm{a}\end{array}$ & $\begin{array}{l}65.0 \mathrm{a} \\
84.2 \mathrm{a}\end{array}$ & $\begin{array}{l}6 a b \\
5 \quad b\end{array}$ \\
\hline
\end{tabular}

* Floración: 1 = baja; 9 = alta

** Prueba de Duncan $(\mathrm{p}=0.05)$ 
Esto permitiría separar aquellas con este tipo de comportamiento para formar el material genético que va a ser suministrado al agricultor, para su producción a nivel comercial. Así mismo, se hace necesario evaluar el comportamiento de las progenies durante un mayor número de generaciones a fin de predecir la durabilidad del material genético en el nivel de producción comercial.

Los resultados sobre intensidad de la floración indican que no hubo diferencias significativas entre las generaciones de polinización libre. Hubo diferencias significativas para este carácter entre las progenies estudiadas; no las hubo, sin embargo, entre las generaciones dentro de las progenies. Esto sugiere que la floración no se podría ver afectada con el avance en las generaciones. Es necesario sin embargo, observar este efecto sobre el rendimiento de bayas y de semillas; de la disponibilidad de las semillas va a depender especialmente, la posibilidad de continuar con la siembra de las subsiguientes generaciones.

\section{REFERENCIAS BIBLIOGRÁFICAS}

1. Atlin, G. 1983. Inbreeding in TPS progenies: Implications for breeding and seedproduction strategy. In: Report of the XXVI Planning Conference on "The Presentand Future Strategies for Potato Breeding and Improvement". International Potato Center. Lima, Perú. pp. 71-85.

2. Centro Internacional de la Papa. 1987. Informe Anual del CIP, 1986-1987. Lima, Perú. 232 p.

3. Kidane-Mariam, H.M.; Arndt, G.C.; Macaso-Khwaja, A.C.; Peloquin, S.J. 1985.Comparison between 4X x 2X hybrid and open pollinated truc Potato seed families. Potato Research 28: 35-42.

4. Kidane-Mariam, H.M.; Mendoza, HA.; Wissar, R.O. 1985. Performance of true potato seed families derived from intermating tetraploid parental lines. Am. Potato J. 62: 643652.

5. Krantz, FA. 1946. Polalo Breeding Methods III. A suggested procedure for Potato breeding. University of Minnesota. Tech. Bulletin 73.

6. Macaso-Khwaja, A.C.; Peloquin, S.J. 1983. Tuber yield of families from open-pollinated and hybrid true potato seed. Am. Potato J. 60: 645-651. 
7. Mendoza, HA. 1979. Preliminary results on yield and uniformily of Potatoes grown from true seed. In: Report of a Planning Conference on "Production of Potatoes from True Seed". International Potato Center. Manila, Philippines. pp.156172.

8. Mendoza, HA.; Haynes, F.L. 1973. Some aspects of breeding and inbreeding potatoes. Am. Potato J. 50: 216-222.

9. Thompson, P.G.; Mendoza, HA.; Plaisted, R.L. 1983. Estimation of genetic parameters for characters selected for potato propagation by true seed in an andigena population. Am. Potato J. 60: 393-401. 